\title{
Enhancing Long-Lived Particles Searches at the LHC with Precision Timing Information
}

\author{
Jia Liu, ${ }^{1}$ Zhen Liu, ${ }^{2,3}$ and Lian-Tao Wang ${ }^{1,4}$ \\ ${ }^{1}$ Enrico Fermi Institute, University of Chicago, Chicago, Illinois 60637, USA \\ ${ }^{2}$ Theoretical Physics Department, Fermi National Accelerator Laboratory, Batavia, Illinois 60510, USA \\ ${ }^{3}$ Maryland Center for Fundamental Physics, Department of Physics, University of Maryland, College Park, Maryland 20742, USA \\ ${ }^{4}$ Kavli Institute for Cosmological Physics, University of Chicago, Chicago, Illinois 60637, USA
}

(Received 24 June 2018; revised manuscript received 17 October 2018; published 3 April 2019)

\begin{abstract}
We explore the physics potential of using precision timing information at the LHC in searches for longlived particles (LLPs). In comparison with the light standard model particles, the decay products of massive LLPs arrive at detectors with time delays around the nanosecond scale. We propose new strategies to take advantage of this time delay feature by using initial state radiation to time stamp the collision event and require at least one LLP to decay within the detector. This search strategy is effective for a broad range of models. In addition to outlining this general approach, we demonstrate its effectiveness with the projected reach for two benchmark scenarios: a Higgs boson decaying into a pair of LLPs, and pair production of long-lived neutralinos in the gauge mediated supersymmetry breaking models. Our strategy increases the sensitivity to the lifetime of the LLP by two orders of magnitude or more and particularly exhibits a better behavior with a linear dependence on the lifetime in the large lifetime region compared to traditional LLP searches. The timing information significantly reduces the standard model background and provides a powerful new dimension for LLP searches.
\end{abstract}

DOI: 10.1103/PhysRevLett.122.131801

The presence of long-lived particles (LLPs) can be a striking feature of many new physics models [1-11]. At the same time, vast swaths of the possible parameter space of the LLP remain unexplored by LHC searches. LHC general purpose detectors, ATLAS and CMS, provide full angular coverage and sizable volume, making them ideal for LLP searches. However, searches for LLPs that decay within a few centimeters of the interaction point suffer from large standard model (SM) backgrounds. LLPs produced at the LHC generically travel slower than the SM background and decay at macroscopic distances away from the interaction point. Hence, they arrive at outer particle detectors with a sizable time delay.

In this Letter, we focus on a general strategy that uses precision timing as a tool to suppress SM backgrounds and enhances sensitivity to LLPs at the LHC. Recently, precision timing upgrades with a timing resolution of 30 picoseconds have been proposed to reduce pileup for the upcoming runs with higher luminosity, including a MIP timing detector (MTD) [12] by the CMS collaboration for the barrel and end cap region in front of the electromagnetic calorimeter, the high granularity timing detector [13] by the ATLAS

Published by the American Physical Society under the terms of the Creative Commons Attribution 4.0 International license. Further distribution of this work must maintain attribution to the author(s) and the published article's title, journal citation, and DOI. Funded by SCOAP ${ }^{3}$. collaboration in end cap and forward region, and similarly multiple precision timing upgrades [14] by the LHCb collaboration. The usage of (less precise) timing information for long-lived particle searches has been discussed in the past and applied to a very limited class of signals [15]. In this Letter, as a strategy applicable to a broad range of models, we propose the use of a generic initial state radiation (ISR) jet to time stamp the hard collision and require only a single LLP decay inside the detector with a significant time delay. Such a strategy can greatly suppress the SM background and reach a sensitivity two orders of magnitude or more better than traditional searches in a much larger parameter space [6,27-29].

With a general triggering and search strategy that can capture most LLP decays, we show a striking improvement in sensitivity and coverage for LLPs. In addition to the MTD at CMS, we also consider a hypothetical timing layer on the outside of the ATLAS muon spectrometer (MS) as an estimate of the best achievable reach of our proposal for LLPs with long lifetimes [30].

Basics of timing.-While particle identification and kinematic reconstruction are highly developed, usage of timing information has so far been limited since prompt signatures are often assumed. Such an assumption could miss a crucial potential signature of an LLP, a significant time delay. Here we outline a general beyond standard model (BSM) signal search strategy that uses the timing information and the corresponding background consideration. A typical signal event of LLP is shown in Fig. 1. An LLP, denoted as $X$, travels a distance $\ell_{X}$ into a detector 


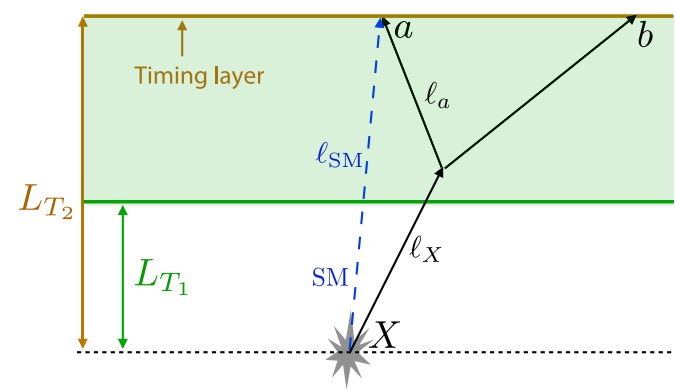

FIG. 1. An event topology with an LLP $X$ decaying into two light SM particles $a$ and $b$. A timing layer, at a transverse distance $L_{T_{2}}$ away from the beam axis (horizontal gray dotted line), is placed at the end of the detector volume (shaded region). The trajectory of a reference SM background particle is also shown (blue dashed line). The gray polygon indicates the primary vertex.

volume and decays into two light SM particles $a$ and $b$, which then reach timing detector at a transverse distance $L_{T_{2}}$ away from the beam axis. Typically, the SM particles travel at velocities close to the speed of light. For simplicity, we consider neutral LLP signals where background from charged particles can be vetoed using particle identification and isolation. The decay products of $X$ arrive at the timing layer with a time delay

$$
\Delta t_{\text {delay }}^{i}=\frac{\ell_{X}}{\beta_{X}}+\frac{\ell_{i}}{\beta_{i}}-\frac{\ell_{\mathrm{SM}}}{\beta_{\mathrm{SM}}},
$$

for $i$ th decay products from $X$ and $\beta_{i} \simeq \beta_{\mathrm{SM}} \simeq 1$. It is necessary to have prompt particles from production or decay, or ISR, which arrives at timing layer with the speed of light, to derive the time of the hard collision at the primary vertex (to "time stamp" the hard collision).

In Fig. 2, we show typical time delay $\Delta t$ distribution for the CMS MTD for benchmark signals and the backgrounds. The two benchmark signals considered here are the glueballs from Higgs boson decays, and the neutralino and chargino pair production in the gauge mediated SUSY breaking (GMSB) scenario [2,3]. Both the glueballs and lightest neutralino proper lifetimes are set to have $c \tau=10 \mathrm{~m}$. The $10 \mathrm{GeV}$ glueballs have larger average boost comparing to the $50 \mathrm{GeV}$ glueballs, and hence have a sizable fraction of the signals with delays less than $1 \mathrm{~ns}$. The GMSB signal is not boosted and hence significantly delayed compared to the backgrounds, with more than $70 \%$ of the signal having $\Delta t>1 \mathrm{~ns}$.

Search strategy.-We consider events with at least one ISR jet to time stamp the PV and one delayed SM object coming from the LLP decay. We propose two searches using the time delay information:

\begin{tabular}{lccccccc}
\hline & $L_{T_{2}}$ & $L_{T_{1}}$ & Trigger & $\epsilon_{\text {trig }}$ & $\epsilon_{\text {sig }}$ & $\epsilon_{\text {fake }}^{j}$ & Ref. \\
\hline MTD & $1.17 \mathrm{~m}$ & $0.2 \mathrm{~m}$ & DelayJet & 0.5 & 0.5 & $10^{-3}$ & {$[12]$} \\
MS & $10.6 \mathrm{~m}$ & $4.2 \mathrm{~m}$ & MS ROI & $0.25,0.5$ & 0.25 & $5 \times 10^{-9}$ & {$[27]$}
\end{tabular}

The size of the detector volume is described by transverse distance to the beam pipe from $L_{T_{1}}$ to $L_{T_{2}}$, where $L_{T_{2}}$ is the

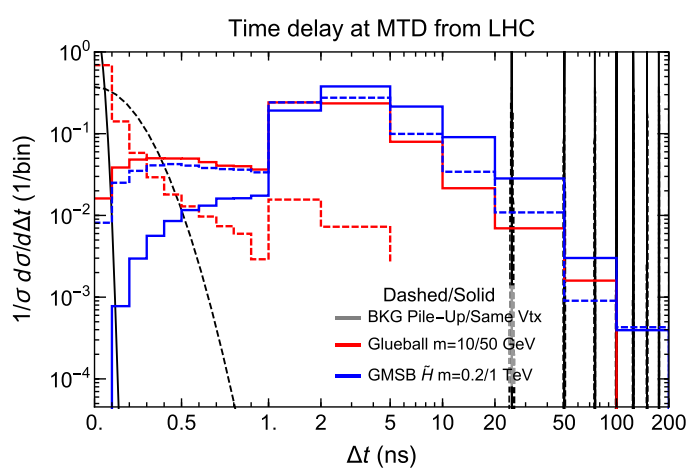

FIG. 2. The differential $\Delta t$ distribution for typical signals and backgrounds at $13 \mathrm{TeV}$ LHC. The plot is normalized to the fraction of events per bin with varying bin sizes, in linear $(\Delta t<1 \mathrm{~ns})$ and logarithmic scale $(>1 \mathrm{~ns})$, respectively. Two representative signal models are shown with different masses. The LLP proper lifetime is set to $10 \mathrm{~m}$, and the distribution only counts events decayed within $\left[L_{T_{1}}, L_{T_{2}}\right]$ of $[0.2,1.17] \mathrm{m}$ in the transverse direction, following the geometry of the CMS MTD in the barrel region. For the background distribution shown in gray curves, we assume bunch spacing of $25 \mathrm{~ns}$. The solid and dashed gray curves represent backgrounds from the same hard collision vertex and hence with a precision timing uncertainty of $\delta_{t}^{\mathrm{PT}}=30 \mathrm{ps}$ and from the pileup with a spread of $\delta_{t}=190 \mathrm{ps}$, respectively.

timing layer location and $L_{T_{1}}$ is the minimal displacement requirement for a analysis. For both searches, we assume a similar timing resolution of $30 \mathrm{ps.} \mathrm{For} \mathrm{the} \mathrm{MS} \mathrm{search,}$ because of the larger time delay and much less background due to "shielding" by inner detectors, a time resolution of $0.2-2$ ns could achieve a similar physics reach. The $\epsilon_{\text {trig }}, \epsilon_{\text {sig }}$, and $\epsilon_{\text {fake }}^{j}$ are the efficiencies for trigger, signal selection, and a QCD jet faking the delayed jet signal with $p_{T}>30 \mathrm{GeV}$ in MTD and MS searches, respectively.

For the MTD search, we assume a new trigger strategy dubbed "DelayJet" using precision timing information at CMS. This can be realized by putting a minimal time delay cut when comparing the prompt time stamping jet (with $p_{T}>30 \mathrm{GeV}$ ) with the arrival time of another jet (with $p_{T}>30 \mathrm{GeV}$ ) at the timing layer. In Supplemental Material section (d) [15,30], we describe some of the recent effort by the experimental collaboration to implement this in the triggering upgrade.

The MTD signal, after requiring $L_{T_{1}}$ of $0.2 \mathrm{~m}$, will not have good tracks associated with it. Hence, the major SM background is from trackless jets. The jet fake rate of $\epsilon_{\text {fake }}^{\mathrm{j}, \mathrm{MTD}}=10^{-3}$ is estimated using PYTHIA [42] by simulating the jets with minimal $p_{T}$ of $30 \mathrm{GeV}$ and study the anti-kt jets with $R=0.4$, where all charged constituent hadrons are too soft $\left(p_{T}<1 \mathrm{GeV}\right)$. For comparison with other studies, see the Supplemental Material section (c) $[15,30]$.

For the MS search, we use the MS region of interest (MS ROI) trigger from a very similar search [43] as a reference, with an efficiency of $\epsilon_{\text {trig }}=0.25$ and 0.5 for the two 
benchmark BSM signals, and a signal selection efficiency of $\epsilon_{\text {sig }}=0.25$. The backgrounds are mainly from the punch-through jets, and its fake efficiency can be inferred to be $\epsilon_{\text {fake }}^{\mathrm{j}, \mathrm{MS}}=5.2 \times 10^{-9}$, normalized to 1300 fake MS barrel events at $8 \mathrm{TeV}$ [43], see details in the Supplemental Material section (c) $[15,30]$.

Background consideration.-The main sources of the SM background faking the delayed and displaced signal are from jets or similar hadronic activities. The origin of background can be classified into same-vertex (SV) hard collision and pileup (PU). For this Letter, we assume the time-spread distributions follow a Gaussian distribution.

$$
\frac{d \mathcal{P}(\Delta t)}{d \Delta t}=\frac{1}{\sqrt{2} \delta_{t}} E^{-\left(\Delta t^{2} / 2 \delta_{t}^{2}\right)},
$$

where the time spreads $\delta_{t}$ differ for different sources of backgrounds. The validity of these description should be scrutinized by experimental measurement, e.g., from zerobias events. From Refs. [12,16-18], the Gaussian description is appropriate up to a probability of a $10^{-4}$ to $10^{-6}$ level. Even in the case the Gaussian fails at the tail, a suppression power of $10^{-5}$ is already enough for MS. For MTD, one can require two time delayed objects to double the Gaussian suppression. Since the time delay is dominated by slow movement of $X$, the two jets from $X$ decay satisfy this requirement easily.

The SV background mainly comes from QCD multijet production. At least one prompt jet is required to time stamp the event, while another trackless jet from the same hard collision fakes long-lived signals. The fake jet has an intrinsic time delay $\Delta t=0$. However, it spreads out in time due to finite timing resolution, $\delta_{t}^{\mathrm{PT}}=30 \mathrm{ps}$. At $13 \mathrm{TeV}$ with $\mathcal{L}_{\text {int }}=3 \mathrm{ab}^{-1}$, the estimated number of background events are

$$
\begin{gathered}
\text { MTD: } N_{\text {bkg }}^{\mathrm{SV}}=\sigma_{\mathrm{j}} \mathcal{L}_{\text {int }} \epsilon_{\text {trig }}^{\mathrm{MTD}} \epsilon_{\text {fake }}^{j, \mathrm{MTD}} \approx 1 \times 10^{11,}, \\
\mathrm{MS}: N_{\text {bkg }}^{\mathrm{SV}}=\sigma_{\mathrm{j}} \mathcal{L}_{\text {int }} \epsilon_{\text {trig }}^{\mathrm{MS}} \epsilon_{\text {fake }}^{j, \mathrm{MS}} \approx 4 \times 10^{5},
\end{gathered}
$$

where $\sigma_{\mathrm{j}} \simeq 1 \times 10^{8}, \mathrm{pb}$ is the multijets cross section with two jets $p_{T}^{j}>30 \mathrm{GeV}$, and $\epsilon_{\text {trig }}$ and $\epsilon_{\text {fake }}^{j}$ are the trigger and fake-rate efficiencies without using timing information.

The PU background contains two hard collisions within the same bunch crossing that do not occur at the same time. The PU background requires the coincidence of a triggered hard event and a fake signal event from pileup collision whose primary vertex fails to be reconstructed. At the HL LHC, the total number of background events can be estimated,

$$
\begin{gathered}
\text { MTD: } N_{\text {bkg }}^{\mathrm{PU}}=\sigma_{\mathrm{j}} \mathcal{L}_{\text {int }} \epsilon_{\text {trig }}^{\mathrm{MTD}}\left(\bar{n}_{\mathrm{PU}} \frac{\sigma_{\mathrm{j}}}{\sigma_{\text {inc }}} \epsilon_{\text {fake }}^{j, \mathrm{MTD}} f_{\mathrm{nt}}^{j}\right) \approx 2 \times 10^{7}, \\
\mathrm{MS}: N_{\mathrm{bkg}}^{\mathrm{PU}}=\sigma_{\mathrm{j}} \mathcal{L}_{\text {int }} \epsilon_{\text {trig }}^{\mathrm{MS}}\left(\bar{n}_{\mathrm{PU}} \frac{\sigma_{\mathrm{j}}}{\sigma_{\text {inc }}} \epsilon_{\text {fake }}^{j, \mathrm{MS}} f_{\mathrm{nt}}^{j}\right) \approx 50,
\end{gathered}
$$

where $\sigma_{\text {inc }}=80 \mathrm{mb}$ is the inelastic proton-proton cross section at $13 \mathrm{TeV}$ [44]. $\bar{n}_{\mathrm{PU}} \approx 100$ (nominally 140 or 200 [45]) is the average number of inelastic interactions per bunch crossing at the HL LHC. In Eq. (4), one hard collision needs to time stamp the event, while the other hard collision contains at least two jets, all of which have to be neutral to miss the primary vertex reconstruction. Otherwise, this second hard collision will leave tracks and be reconstructed as another vertex in the tracker, thus getting vetoed. Therefore, the background $N_{\mathrm{bkg}}^{\mathrm{PU}}$ is suppressed by at least one additional factor of neutral jet fraction $f_{\mathrm{nt}}^{j} \simeq 10^{-3}$. This additional factor $f_{\mathrm{nt}}^{j}$, more strictly speaking, should be the probability for a multijet process whose primary vertex fails to be reconstructed and misassigned to the triggered vertex, which need to be estimated through a full detector simulation and calibrated with data.

The key difference between the PU and SV backgrounds is that the time spread being determined by the beam property for the former (190 ps [12]), and by the timing resolution for the latter (30 ps [12]). For the MTD (MS) search, if we apply cut $\Delta t>1(0.4) \mathrm{ns}$, the total estimated events from a SM background is $1.3(0.86)$, where the SV background become completely negligible.

Backgrounds not from the hard collision have larger temporal spread, such as cosmic ray, beam halo, misconnected tracks, interaction with detector material, etc. At the same time, their properties are well measured and can be vetoed effectively. For example, for the MS search, displaced vertex reconstruction can help suppress the above backgrounds. Its efficiency has been included in $\epsilon_{\text {sig }}$ [27]. In another example, the nonpointing photon searches study at ATLAS [18] found that such backgrounds are negligible, with two photon final states which only have directional information. Reference [19] measured the stopped particle signatures and found that the energy cut alone can reduce the background to single digit. In comparison, our signal has more kinematic features, such as large energy deposition (more than $30 \mathrm{GeV}$ ) and high track multiplicities with sizable time delay. It can be further separated from these backgrounds. The argument for MS also applies to the CMS MTD search. The search for a pair of jets from one displaced vertex [28] found the SM QCD background to be dominant. Moreover, since the MTD detector is much smaller than MS, the cosmic ray background is less problematic. Even assuming the number of SM background events to be 100, the limits in Fig. 3 are only weakened by a factor of 5 .

Augmented sensitivity on LLPs through precision timing.-Our first example, Signal A (SIGA), is a Higgs boson decaying to glueballs with subsequent decays into SM jet pairs. This occurs in a model [10] where the Higgs boson is the portal to a dark QCD sector whose lightest states are the long-lived glueballs. Typical energy of the glueball is set by the Higgs mass, and the time delay depends on glueball mass. 


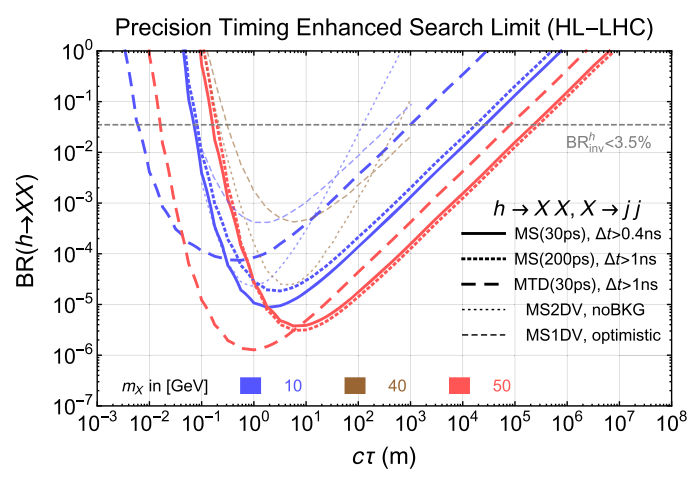

FIG. 3. The $95 \%$ C.L. limit on $\mathrm{BR}(h \rightarrow X X)$ for signal process $p p \rightarrow j h$ with subsequent decay $h \rightarrow X X$ and $X \rightarrow j j$. Different colors indicate different masses of the particle $X$. The thick solid and dotted (thick long dashed) lines indicate MS (MTD) searches with different timing cuts. The numbers in parentheses are the assumed timing resolutions. Other $13 \mathrm{TeV}$ LHC projections $[29,51]$ are plotted in thin lines.

The second example, Signal B (SIGB), is the decay of the lightest neutralino in the GMSB scenario. Its decay into SM bosons $(Z, \mathrm{~h}$, or $\gamma)$ and gravitino is suppressed by the SUSY breaking scale $\sqrt{F}$, and it can be naturally longlived. This benchmark represents the timing behavior of pair produced particles at the LHC without an intermediate resonance.

For both examples, time stamping the hard collision is achieved by using an ISR jet:

$$
\begin{array}{ll}
\text { SIGA: } p p \rightarrow h+j, & h \rightarrow X+X, \quad X \rightarrow \mathrm{SM}, \\
\mathrm{SIGB}: p p \rightarrow \tilde{\chi} \tilde{\chi}+j, & \tilde{\chi}_{1}^{0} \rightarrow h+\tilde{G} \rightarrow \mathrm{SM}+\tilde{G} .
\end{array}
$$

For SIGB, other electroweakinos $\tilde{\chi}$, such as charginos $\tilde{\chi}^{ \pm}$or heavier neutralino $\tilde{\chi}_{2}^{0}$, promptly decay into the lightest neutralino state $\tilde{\chi}_{1}^{0}$ plus soft particles. Hence, we take the inclusive Higgsino pair production cross section for this process.

To emphasize the power of timing, we rely mostly on the timing information to suppress background and make only minimal cuts. We only require one low $p_{T}$ ISR jet, with $p_{T}^{j}>30 \mathrm{GeV}$ and $\left|\eta_{j}\right|<2.5$. In both signal benchmarks, we require at least one LLP decays inside the detector. We generate signal events using MADGRAPH5 [46] at the parton level and adopt the UFO model file from [47] for the GMSB simulation. After detailed simulations of the delayed arrival time, we derive the projected sensitivity to SIGA and SIGB using the cross sections obtained in Ref. [48] and Refs. [49,50], respectively.

For SIGA, the 95\% C.L. sensitivity is shown in Fig. 3. We assume $X$ decays to SM jet pairs with $100 \%$ branching fraction. The MTD and MS searches, with 30 ps timing resolution, are plotted in thick dashed and solid lines, respectively. For MS, the best reach of $\operatorname{BR}(h \rightarrow X X)$ is about a few $10^{-6}$ for $c \tau<10 \mathrm{~m}$. It is relatively insensitive to the mass of $X$ when $m_{X}>10 \mathrm{GeV}$ because $X$ are moving slowly enough to pass the timing cut. For the MS search, a less precise timing resolution (200 ps) has also been considered with cut $\Delta t>1 \mathrm{~ns}$. After the cut, the backgrounds from SV and PU for MS search are 0.11 and $7.0 \times 10^{-3}$ respectively, and the SV background dominates. The reach for heavy $X$ is almost not affected, while reduced by a factor of $\sim 2$ for light $X$.

In Fig. 3, we compare MTD and MS (thick lines) with $13 \mathrm{TeV}$ HL LHC (with $3 \mathrm{ab}^{-1}$ integrated luminosity) projections, two displaced vertex (DV) at MS using zero background assumption (thin dotted) and one DV at MS using a data-driven method with optimistic background estimation (thin dashed) from Ref. [29]. The projected limits from invisible Higgs decay at $13 \mathrm{TeV}$ [51] is also shown in Fig. 3.

For SIGB, we show the projected 95\% C.L. exclusion reach in the plane of Higgsino mass $m_{\tilde{\chi}}$ and proper lifetime $c \tau$ in Fig. 4. The projected coverage of the MTD and MS searches in blue and red shaded regions, respectively. Due to the slow motion of $\tilde{\chi}$, we show the projections with a tight (solid lines) and a loose (dashed lines) $\Delta t$ requirement. The loose selection, $\Delta t>10 \mathrm{~ns}$ allows us to use the current muon timing resolution of $2 \mathrm{~ns}$ [52] to achieve similar coverage. Although MTD and MS searches with $\Delta t>1$ and 0.4 ns cuts have background event of order 1 , we also show the sensitivity reach with a sizable background of 100 at the HL LHC. We observe a similar behavior for the coverage of MTD and MS searches in term of the lifetime for SIGB.

Furthermore, we draw gray dashed-dotted lines for SUSY breaking scale $\sqrt{F}$. To compare with existing LLP searches and their projection, we follow Ref. [6] and quote the most sensitive CMS displaced dijet search conducted at $8 \mathrm{TeV}[28]$, and show the projected sensitivity

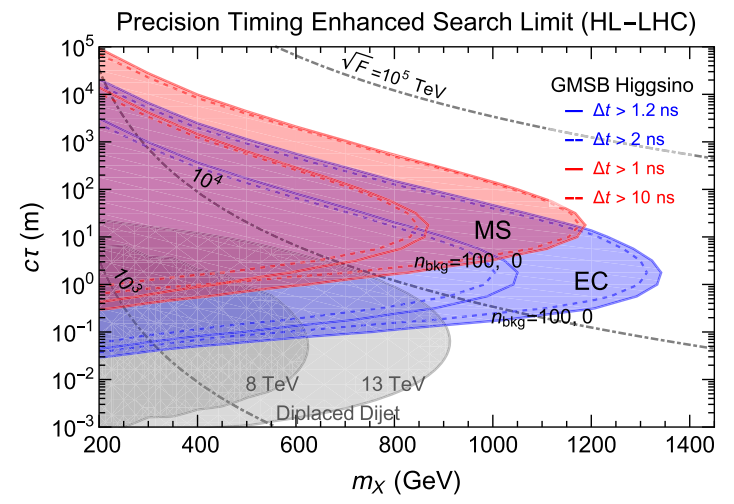

FIG. 4. The projected 95\% C.L. limit on the Higgsino masslifetime plane for signal process of Higgsino pair production in association with jets, with subsequent decay of the lightest Higgsino $\tilde{\chi}^{0} \rightarrow h \tilde{G}$ and $h \rightarrow b b$ in GMSB scenario. We decouple other electroweakinos and have Higgsino-like chargino $\tilde{\chi}^{ \pm}$and neutralino $\tilde{\chi}_{2}^{0}$ nearly degenerate with $\tilde{\chi}_{1}^{0}$. 
at $13 \mathrm{TeV}$ HL LHC assuming statistical dominance for the background. We can see timing searches almost double the reach of $m_{\tilde{\chi}}$ with lifetime around one meter, and extend the sensitivity to very long lifetime, up to $10^{5} \mathrm{~m}$ for a $200 \mathrm{GeV}$ LLP.

Discussion.-We demonstrate in this Letter that exploiting timing information can significantly enhance the sensitivities of LLP searches at the LHC. To emphasize the advantage of timing, we made minimal requirements on the signal, with one ISR jet and a delayed signal. Further optimization can be developed for more dedicated searches. The time stamping ISR jet can be replaced by other objects, such as leptons or photons. Depending on the underlying signal and model parameters, one can also use prompt objects from signal production and decay. In addition, for specific searches, one could also optimize the selection of the signal based on the decay products of LLPs. Finally, we emphasize that the current LLP searches are complementary to the timing proposed in this Letter. Once combined, the current searches should in general gain better sensitivity for heavy LLP.

[1] R. Barbier et al., R-parity violating supersymmetry, Phys. Rep. 420, 1 (2005).

[2] G. F. Giudice and R. Rattazzi, Theories with gauge mediated supersymmetry breaking, Phys. Rep. 322, 419 (1999).

[3] P. Meade, M. Reece, and D. Shih, Long-lived neutralino NLSPs, J. High Energy Phys. 10 (2010) 067.

[4] A. Arvanitaki, N. Craig, S. Dimopoulos, and G. Villadoro, Mini-split, J. High Energy Phys. 02 (2013) 126.

[5] N. Arkani-Hamed, A. Gupta, D. E. Kaplan, N. Weiner, and T. Zorawski, Simply unnatural supersymmetry, arXiv:1212.6971.

[6] Z. Liu and B. Tweedie, The fate of long-lived superparticles with hadronic decays after LHC Run 1, J. High Energy Phys. 06 (2015) 042.

[7] Z. Chacko, H.-S. Goh, and R. Harnik, The Twin Higgs: Natural Electroweak Breaking from Mirror Symmetry, Phys. Rev. Lett. 96, 231802 (2006).

[8] G. Burdman, Z. Chacko, H.-S. Goh, and R. Harnik, Folded supersymmetry and the LEP paradox, J. High Energy Phys. 02 (2007) 009.

[9] J. Kang and M. A. Luty, Macroscopic strings and "Quirks" at colliders, J. High Energy Phys. 11 (2009) 065.

[10] N. Craig, A. Katz, M. Strassler, and R. Sundrum, Naturalness in the dark at the LHC, J. High Energy Phys. 07 (2015) 105.

[11] A. Davoli, A. De Simone, T. Jacques, and V. Sanz, Displaced vertices from pseudo-dirac dark matter, J. High Energy Phys. 11 (2017) 025.

[12] Technical Proposal for a Mip Timing Detector in the CMS Experiment Phase 2 UPGRADE, Technical Report No. CERN-LHCC-2017-027, LHCC-P-009, CERN, Geneva, 2017, https://cds.cern.ch/record/2296612. This document describes a MIP timing detector for the Phase2 upgrade of the CMS experiment, in view of HL-LHC running.
[13] C. Allaire et al., Beam test measurements of low gain avalanche detector single pads and arrays for the ATLAS high granularity timing detector, J. Instrum. 13, P06017 (2018).

[14] R. e. a. Aaij (LHCb Collaboration), Expression of Interest for a Phase-II LHCb Upgrade: Opportunities in flavour physics, and beyond, in the HL-LHC era, Technical Report No. CERN-LHCC-2017-003, CERN, Geneva, 2017, http:// cds.cern.ch/record/2244311.

[15] See Supplemental Material at http://link.aps.org/ supplemental/10.1103/PhysRevLett.122.131801 for a more detailed discussion and references therein [16-26].

[16] S. Chatrchyan et al. (CMS Collaboration), Searches for long-lived charged particles in $p p$ collisions at $\sqrt{s}=7$ and 8 TeV, J. High Energy Phys. 07 (2013) 122.

[17] S. Chatrchyan et al. (CMS Collaboration), Search for heavy long-lived charged particles in $p p$ collisions at $\sqrt{s}=7 \mathrm{TeV}$, Phys. Lett. B 713, 408 (2012).

[18] G. Aad et al. (ATLAS Collaboration), Search for nonpointing and delayed photons in the diphoton and missing transverse momentum final state in $8 \mathrm{TeV} p p$ collisions at the LHC using the ATLAS detector, Phys. Rev. D 90, 112005 (2014).

[19] A. M. Sirunyan et al. (CMS Collaboration), Search for decays of stopped exotic long-lived particles produced in proton-proton collisions at $\sqrt{s}=13 \mathrm{TeV}$, J. High Energy Phys. 05 (2018) 127.

[20] M. Aaboud et al. (ATLAS Collaboration), Search for metastable heavy charged particles with large ionization energy loss in pp collisions at $\sqrt{s}=13 \mathrm{TeV}$ using the ATLAS experiment, Phys. Rev. D 93, 112015 (2016).

[21] D. A. Toback and P. Wagner, Prospects of searches for neutral, long-lived particles which decay to photons using timing at CDF, Phys. Rev. D 70, 114032 (2004).

[22] M. Goncharov et al., The timing system for the CDF electromagnetic calorimeters, Nucl. Instrum. Methods Phys. Res., Sect. A 565, 543 (2006).

[23] A. Abulencia et al. (CDF Collaboration), Search for Heavy, Long-Lived Particles that Decay to Photons at CDF II, Phys. Rev. Lett. 99, 121801 (2007).

[24] T. Aaltonen et al. (CDF Collaboration), Search for heavy, long-lived neutralinos that decay to photons at CDF II using photon timing, Phys. Rev. D 78, 032015 (2008).

[25] T. Aaltonen et al. (CDF Collaboration), Signaturebased search for delayed photons in exclusive photon plus missing transverse energy events from $p \bar{p}$ collisions with $\sqrt{s}=1.96 \mathrm{TeV}$, Phys. Rev. D 88, 031103 (2013).

[26] CMS Collaboration, Search for long-lived neutral particles in the final state of delayed photons and missing energy in proton-proton collisions at $\sqrt{s}=8 \mathrm{TeV}$, Technical Report No. CMS-PAS-EXO-12-035, CERN, Geneva, 2015, http:// cds.cern.ch/record/2063495.

[27] G. Aad et al. (ATLAS Collaboration), Search for long-lived, weakly interacting particles that decay to displaced hadronic jets in proton-proton collisions at $\sqrt{s}=8 \mathrm{TeV}$ with the ATLAS detector, Phys. Rev. D 92, 012010 (2015).

[28] V. Khachatryan et al. (CMS Collaboration), Search for longlived neutral particles decaying to quark-antiquark pairs in proton-proton collisions at $\sqrt{s}=8 \mathrm{TeV}$, Phys. Rev. D 91, 012007 (2015). 
[29] A. Coccaro, D. Curtin, H. J. Lubatti, H. Russell, and J. Shelton, Data-driven model-independent searches for longlived particles at the LHC, Phys. Rev. D 94, 113003 (2016).

[30] See Supplemental Material at http://link.aps.org/ supplemental/10.1103/PhysRevLett.122.131801 for more details of our study, including the signal benchmark considerations, the time delay distributions at ATLAS MS, QCD background explanations, and trigger discussion, and references therein [31-41].

[31] B. Andersson, G. Gustafson, G. Ingelman, and T. Sjostrand, Parton fragmentation and string dynamics, Phys. Rep. 97, 31 (1983).

[32] G. Aad et al. (ATLAS Collaboration), Jet energy measurement with the ATLAS detector in proton-proton collisions at $\sqrt{s}=7 \mathrm{TeV}$, Eur. Phys. J. C 73, 2304 (2013).

[33] M. Klimek, Tagging boosted objects with timing detectors, 2018, https://indico.fnal.gov/event/16151/session/9/ contribution/75/material/slides/0.pdf.

[34] ATLAS Collaboration, Search for pair produced long-lived neutral particles decaying in the ATLAS hadronic calorimeter in $p p$ collisions at $\sqrt{s}=8 \mathrm{TeV}, \mathrm{CERN}$, Geneva, Technical Report No. ATLAS-CONF-2014-041, 2014, https://cds.cern.ch/record/1740972.

[35] R. E. Shrock, A Test for the Existence of Effectively Stable Neutral Heavy Leptons, Phys. Rev. Lett. 40, 1688 (1978).

[36] E. Gallas et al. (FMMF Collaboration), Search for neutral weakly interacting massive particles in the Fermilab Tevatron wide band neutrino beam, Phys. Rev. D 52, 6 (1995).

[37] J. R. Ellis, A. R. Raklev, and O. K. Oye, Gravitino dark matter scenarios with massive metastable charged sparticles at the LHC, J. High Energy Phys. 10 (2006) 061.

[38] J. R. Ellis, A. R. Raklev, and O. K. Oye, Measuring massive metastable charged particles with ATLAS RPC timing information, CERN Reports No. ATL-PHYS-PUB-2007016 and No. ATL-COM-PHYS-2006-093, 2016, http://cds .cern.ch/record/1006573.

[39] S. Banerjee, G. Bélanger, B. Bhattacherjee, F. Boudjema, R. M. Godbole, and S. Mukherjee, Novel signatures for long-lived particles at the LHC, Phys. Rev. D 98, 115026 (2018).

[40] G. Aad et al. (ATLAS Collaboration), Triggers for displaced decays of long-lived neutral particles in the ATLAS detector, J. Instrum. 8, P07015 (2013).

[41] Y. Gershtein, CMS Hardware track trigger: New opportunities for long-lived particle searches at the HL-LHC, Phys. Rev. D 96, 035027 (2017).
[42] T. Sjostrand, S. Mrenna, and P.Z. Skands, A brief introduction to PYTHIA 8.1, Comput. Phys. Commun. 178, 852 (2008).

[43] M. Aaboud et al. (ATLAS Collaboration), Search for longlived, massive particles in events with displaced vertices and missing transverse momentum in $\sqrt{s}=13 \mathrm{TeV} p p$ collisions with the ATLAS detector, Phys. Rev. D 97, 052012 (2018).

[44] M. Aaboud et al. (ATLAS Collaboration), Measurement of the Inelastic Proton-Proton Cross Section at $\sqrt{s}=13 \mathrm{TeV}$ with the ATLAS Detector at the LHC, Phys. Rev. Lett. 117, 182002 (2016).

[45] G. Apollinari, I. Béjar Alonso, O. Brüning, M. Lamont, and L. Rossi, High-Luminosity Large Hadron Collider (HL-LHC): Preliminary Design Report, CERN Yellow Reports: Monographs, CERN, Geneva, 2015, https://cds .cern.ch/record/2116337.

[46] J. Alwall, R. Frederix, S. Frixione, V. Hirschi, F. Maltoni, O. Mattelaer, H. S. Shao, T. Stelzer, P. Torrielli, and M. Zaro, The automated computation of tree-level and next-to-leading order differential cross sections, and their matching to parton shower simulations, J. High Energy Phys. 07 (2014) 079.

[47] N. D. Christensen, P. de Aquino, N. Deutschmann, C. Duhr, B. Fuks, C. Garcia-Cely, O. Mattelaer, K. Mawatari, B. Oexl, and Y. Takaesu, Simulating spin- $\frac{3}{2}$ particles at colliders, Eur. Phys. J. C 73, 2580 (2013).

[48] N. Greiner, S. Höche, G. Luisoni, M. Schönherr, J.-C. Winter, and V. Yundin, Phenomenological analysis of Higgs boson production through gluon fusion in association with jets, J. High Energy Phys. 01 (2016) 169.

[49] B. Fuks, M. Klasen, D. R. Lamprea, and M. Rothering, Gaugino production in proton-proton collisions at a centerof-mass energy of $8 \mathrm{TeV}$, J. High Energy Phys. 10 (2012) 081.

[50] B. Fuks, M. Klasen, D. R. Lamprea, and M. Rothering, Precision predictions for electroweak superpartner production at hadron colliders with Resummino, Eur. Phys. J. C 73, 2480 (2013).

[51] C. Bernaciak, T. Plehn, P. Schichtel, and J. Tattersall, Spying an invisible Higgs boson, Phys. Rev. D 91, 035024 (2015).

[52] A. M. Sirunyan et al. (CMS Collaboration), Performance of the CMS muon detector and muon reconstruction with proton-proton collisions at $\sqrt{s}=13 \mathrm{TeV}, \mathrm{J}$. Instrum. 13, P06015 (2018). 